\title{
Measurement of several transition probabilities in singly-ionized krypton
}

\author{
F. Rodríguez, J. A. Aparicio, A. de Castro, J. A. del Val`, V. R. González, and S. Mar \\ Departamento de Optica y Física Aplicada, Facultad de Ciencias, Universidad de Valladolid, \\ 47071 Valladolid, Spain
}

Received 22 January 2001 / Accepted 20 March 2001

\begin{abstract}
This work reports a collection of 35 transition probabilities of lines in the spectral region $450-580 \mathrm{~nm}$, all of them measured in an emission experiment. Relative intensity measurements have been made in a pulsed discharge lamp and the absolute $A_{k i}$ values have been obtained by using reference data taken from the literature. The electron density has been determined by two-wavelength interferometry and ranges from 0.1 to $0.8 \times 10^{23} \mathrm{~m}^{-3}$ in the plasma. Temperature (14000-24000 K) has been simultaneously determined from three different methods, including the Boltzmann-plot of KrII lines and the KrII/KrI intensities ratios. The final results have been compared with most of the previous existing data.
\end{abstract}

Key words. atomic data - line: profile - plasmas - methods: laboratory

\section{Introduction}

Since 1965, experimental data of transition probabilities of singly-ionized krypton have been obtained. These data should allow researchers to obtain information about atomic structure or the type of coupling to be considered for the theoretical models. However, great discrepancies (Donnelly et al. 1975) exist in the experimental results of the different authors, as well as great uncertainties. In the last ten years there has been a renewed interest in the transition probabilities of KrII. This species has been detected in the spectra of the interstellar medium with help of the Goddard high resolution spectrograph on the Hubble space telescope (Cardelli et al. 1991; Cardelli \& Mayer 1997). Krypton represents the material from which the young early type stars are formed (Leckrone et al. 1993). Moreover, krypton is present in many light source and lasers in laboratory studies and in industrial applications (Graves 1983; Mckee et al. 1996).

Although an important number of experimental works (Levchenko 1971; Miller et al. 1972; Podbiralina et al. 1973; Keil 1973; Samoilov et al. 1975; Baessler et al. 1979; Fonseca \& Campos 1982; Brandt et al. 1982; Bertuccelli \& Di Rocco 1991; Castro et al. 2001) and theoretical works (Koozekanani \& Trusty 1969; El Sherbini 1976;

Send offprint requests to: Dr. J. A. Aparicio,

e-mail: apa@opt.uva.es

* Permanent address: Departamento de Física Aplicada, Universidad de Salamanca, E. Politécnica Superior 05071 Avila, Spain.
Spector \& Garpman 1977; Brandt et al. 1982) have been performed to determine $A_{k i}$-values for KrII, a number of lines in the spectrum exist for which there is no data.

In this work $A_{k i}$-values have been obtained in an emission experiment from measurements performed on a linear discharge lamp, where pure krypton was introduced. The plasma source employed provides not only all kinds of interferometric and spectroscopic recordings with great reproducibility in different discharges, but also makes it possible in a broad range of electron densities ( 0.1 to $\left.0.8 \times 10^{23} \mathrm{~m}^{-3}\right)$ and temperatures $(14000$ to $24000 \mathrm{~K})$. This allows us to acquire reliable spectra for weak isolated and non-isolated lines, very difficult to obtain otherwise. Absolute transition probabilities have been obtained from relative intensity measurements, taking as reference those from Fuhr \& Wiese (1998) and Castro et al. (2001). In this way, the KrII excitation temperature has been determined from the Boltzmann-plot. Other techniques to calculate temperature have also been employed: KrII/KrI intensity ratios and the algorithm described in Gigosos et al. (1994). The very good agreement among these three methods suggests that the plasma is well described by a partial local thermodynamic equilibrium (pLTE) model (van der Mullen 1990). Other experimental cautions, like the existence of self-absorption or spectrometer calibration have been carefully considered. The number of measurements (12) performed for each line, and its very controlled features, allows us to obtain a very good set of

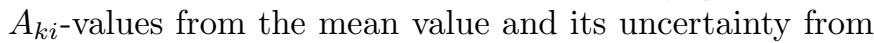
the standard deviation, in a spectral interval where little 
data exist (450-580 nm), as far as we know. These values will be compared with those found in literature.

\section{Experimental arrangement and measurements performed}

Most of the experimental information relative to the plasma source, design and management have already been described by Gigosos et al. (1994), and by del Val et al. (1998). Here we summarize the specific details concerning this experiment. An scheme of the experimental set-up is shown in Fig. 1.

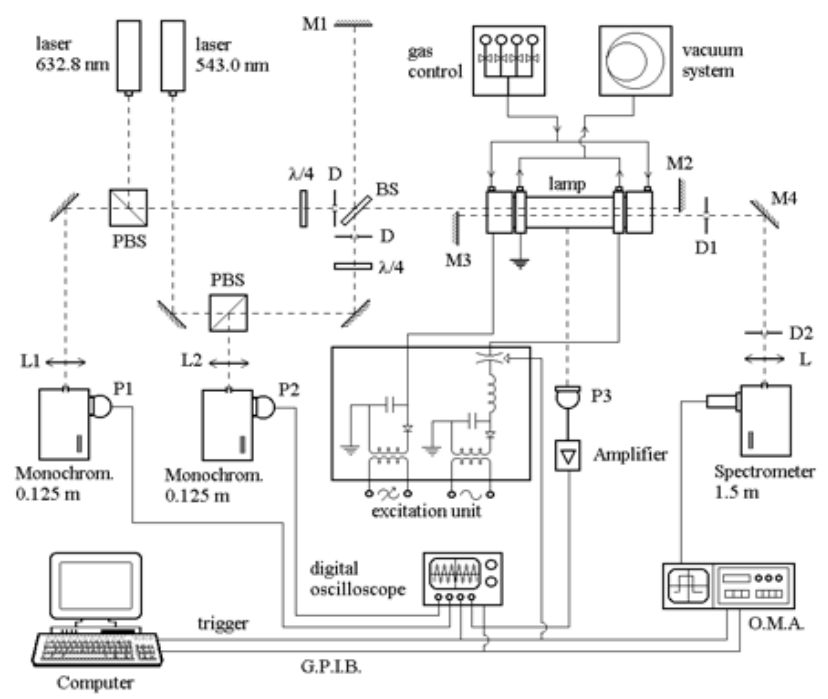

Fig. 1. Experimental arrangement.

The source of plasma consists of a cylindrical tube of Pyrex glass $175 \mathrm{~mm}$ in length and $19 \mathrm{~mm}$ inner diameter. The lamp has been designed to avoid sputtering as much as possible. The plasma was created by discharging a capacitor bank of $20 \mu \mathrm{F}$ charged up to $7.5 \mathrm{KV}$. During the whole experiment the lamp was working with a continuous flow of pure krypton at a rate of $0.82 \mathrm{~cm}^{3} / \mathrm{min}$ and a pressure of $3.3 \times 10^{2} \mathrm{~Pa}$. In these conditions the KrII emission lasts approximately $150 \mu \mathrm{s}$. The gas was pre-ionized in order to obtain the best discharge reliability and the necessary equal initial conditions for the different pulses to be comparable. Spectroscopic and interferometric end-on measurements were made simultaneously during the plasma life, and were taken $2 \mathrm{~mm}$ off the lamp axis, and from symmetric positions relative to it (del Val et al. 1998). According to Fig. 1, the lamp is placed in one of the arms of a Twyman-Green interferometer simultaneously illuminated with two He-Ne lasers $(543.0 \mathrm{~nm}$ and $632.8 \mathrm{~nm})$ in order to determine the electron density evolution curve $N_{\mathrm{e}}(t)$ from the refractivity changes due to free electrons. The spectroscopic beam is directed by two pinholes, $2 \mathrm{~mm}$ diameter (D1, D2), separated $1.5 \mathrm{~m}$ and focused by a cylindrical lens $(\mathrm{L})$ of $150 \mathrm{~mm}$ focal length into the entrance slit of a Jobin-Yvon spectrometer (1.5 m focal length, 1200 lines/mm holographic grating), equipped with an optical multichannel analyzer (O.M.A.). The O.M.A. has a detector array, which is divided into 512 channels (EG\&G 1455R-512-HQ).

After a calibration in wavelength, dispersion was measured to be $12.59 \mathrm{pm} /$ channel at $589.0 \mathrm{~nm}$ at the first order of diffraction with an uncertainty lower than $1 \%$ (Aparicio et al. 1998). A relative intensity calibration of the spectrometer was also very carefully performed. An exhaustive description of the procedures followed can be found in González (1999, 2000). This calibration provides a transmittance function which not only includes the dependence in wavelength of the whole optical system traversed by the spectroscopic beam, but also the different behaviour of the 512 channels of the detector. Its uncertainty has been measured to be lower than $4 \%$.

All measurements were carried out in the first order of diffraction, the same order for which the calibration in wavelength and intensity was performed. Time exposure for the detector was always $5 \mu \mathrm{s}$. Mirror M3, placed behind the plasma column, was used to measure the optical depth and to detect possible self-absorption effects in each line profile. This is detected in any spectral line if the intensities ratio between the spectrum taken without mirror M3 and with it is lower at the peak than at any other part of the profile (González 1999).

As a whole, more than 1000 discharges were performed, corresponding to 8 different spectral intervals. All KrII lines were recorded in 12 different instants of the plasma life, with 10 runs for each instant, five with mirror M3, five without. All measurements were made in the region 450-580 $\mathrm{nm}$. KrII lines were typically registered in the first $150 \mu$ s of plasma life, with the exception of the most intense ones as well as some KrI lines, which were recorded also up to $240 \mu$ s after the discharge. The intensity of the KrI spectral lines increases as the krypton ions recombine. One example of the spectra recorded can be seen in Fig. 2. Concerning the interferometric recordings, 15 interferograms for both laser wavelengths were taken at the end of the experiment, all of them $1 \mathrm{~ms}$ long. They have been used to measure $N_{\mathrm{e}}(t)$.

\section{Data processing and plasma diagnostics}

\subsection{Spectroscopic data processing}

Firstly, for each spectral interval at each instant where measurements were performed, the average spectra of the five runs taken with and without mirror M3 were obtained. Averaged spectra differed from the individual spectra by less than $5 \%$, which gives a good idea of the reproducibility of the plasma source in different pulses. By comparing both averaged spectra and using the algorithms described by González $(1999,2000)$, it has been possible to detect and reconstruct spectral profiles when necessary. It is important to note that self-absorption was detected in less than $10 \%$ of the whole spectral profiles and, in less than $10 \%$ of these cases, the reconstructed profile differed from the measured one without mirror M3 by more than $20 \%$ in 


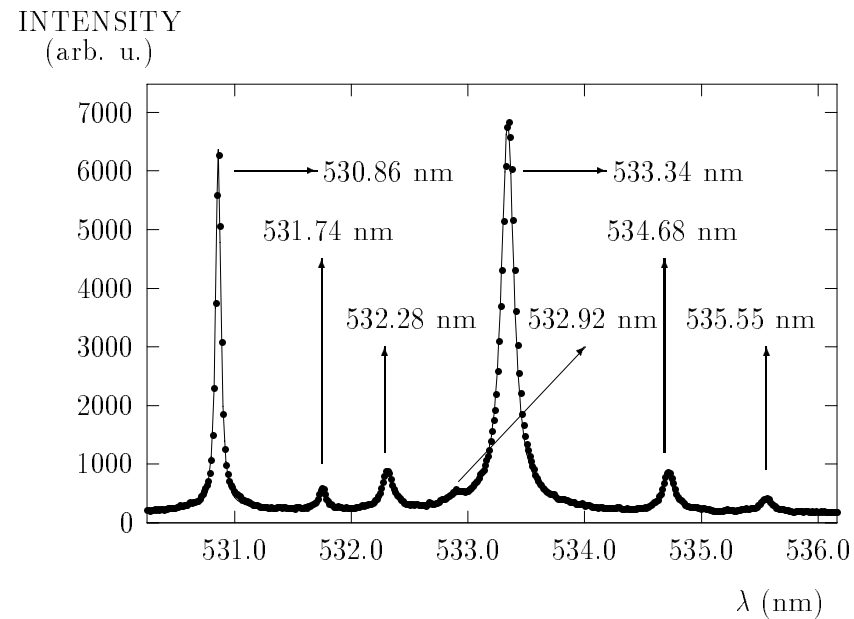

Fig. 2. An example of KrII lines recorded in this experiment.

the peak intensity. These profiles have been rejected from further calculations.

After dividing the averaged spectra by the spectrometer transmittance functions, all of them were fitted to sums of Lorentzian functions plus a luminous background with a linear dependence (Gigosos et al. 1994). This is justified since the Stark effect is the dominant spectral line broadening mechanism at the electron densities achieved in this plasma. Differences between the experimental spectra and the fits were usually lower than $0.5 \%$. The fitting algorithm allows us to determine simultaneously the center, asymmetry, line width and area of each profile. As it can be seen in Fig. 2, even the very overlapped weakest lines have been considered in the fit, not as an objective by themselves, but with the aim of obtaining an accurate measurement of the intensity of their closest isolated spectral profiles. The final uncertainty estimated for the intensity measurement is lower than $15 \%$. This procedures have been apllied to all KrI and KrII lines.

\subsection{Electron density}

Concerning the 15 measured interferometric recordings, they have been processed according to the algorithms developed and described by Aparicio et al. (1998) and de la Rosa et al. (1990). They allow us to obtain for each wavelength an average curve of the phase evolution changes along the plasma life $\Delta \psi_{\lambda_{i}}(t)(i=1,2)$ and from them, the electron density curve $N_{\mathrm{e}}(t)$, according to the expression:

$n_{\mathrm{e}}(t)=\frac{4 \pi \varepsilon_{0} m_{\mathrm{e}} c^{2}}{q_{\mathrm{e}}^{2}} \frac{1}{2 L} \frac{\lambda_{2} \Delta \psi_{\lambda_{1}}(t)-\lambda_{1} \Delta \psi_{\lambda_{2}}(t)}{\lambda_{1}^{2}-\lambda_{2}^{2}}$

$L$ being the plasma column length, which as has usually been demonstrated for this plasma source, is assumed to be the lamp length.

When comparing the $N_{\mathrm{e}}(t)$ curve measured with the two-wavelength method (Eq. (1)) with that obtained at a single wavelength, the differences were always lower than $5 \%$, which indicate the negligible influence of the bound electrons to refractivity changes in this plasma. The electron density curve is shown in Fig. 3, where for each instant, an $10 \%$ error bar has been considered. This is the uncertainty estimated for the electron density in this work.

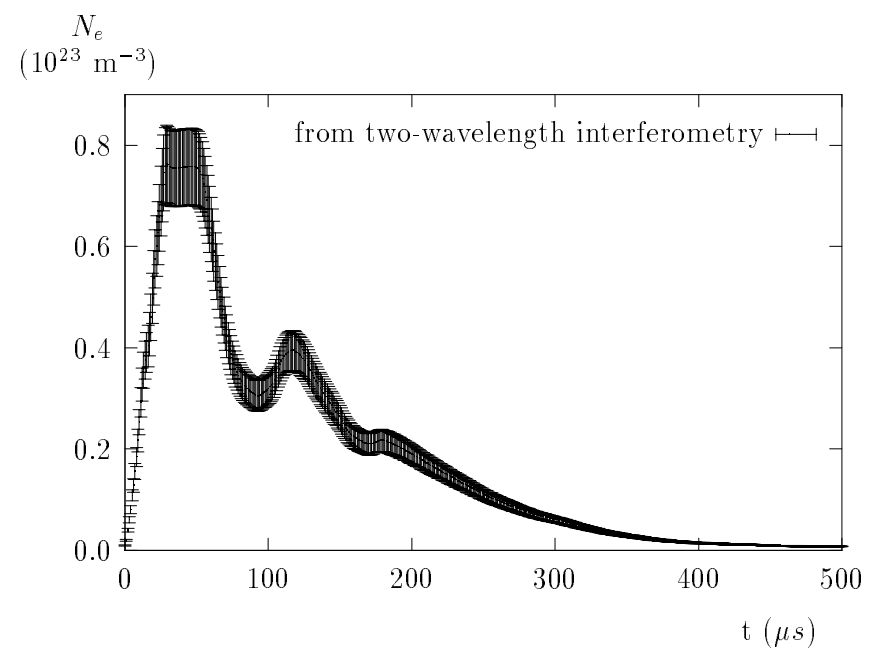

Fig. 3. Electron density evolution curve. An error bar of $10 \%$ has been included to the value obtained at each instant of the plasma life.

\subsection{Temperature}

Relative to temperature measurements, it is a common hypothesis to assume that KrII excitation temperature $T_{\mathrm{KrI}}^{\mathrm{exc}}$, Saha temperature and kinetic electron temperature take similar values in collision-dominated plasmas like those generated in this experiment (van der Mullen 1990). The KrII excitation temperature was obtained from the Boltzmann-plot of some KrII lines, measured in this work, for which the transition probabilities were known. These $A_{k i}$-values were taken from Fuhr \& Wiese (1998) and from Castro et al. (2001). In Table 1, both sets of data are shown and those employed here are labelled with an asterisk. The criterium to select the reference data was to use the data from Castro et al. in all cases except for those lines not measured by them, now measured, and for which Fuhr and Wiese provide data. It is important to note that the values from Castro et al. (2001) were also obtained in an emission experiment by using as a reference the data from Fuhr \& Wiese (1998), so that the whole set of $A_{k i}$-values employed in this work corresponds to the same absolute scale. Once the $A_{k i}$ reference data is selected, a Boltzmann-plot is made for each instant of the plasma life where spectra were taken. Those corresponding to instants $90 \mu$ s and $130 \mu$ s are plotted in Fig. 4. The linear behaviour detected demonstrates that the plasma can be well described by a partial local thermodynamic equilibrium model, at least in the energy level interval considered (16.60-20.86 eV). By using the KrII excitation temperature $T_{\mathrm{KrII}}^{\mathrm{exc}}$ obtained from the slope $b$ of the linear fit 
Table 1. Comparison of the transition probabilities measured from Castro et al. (2001) with the reference employed in their work (Fuhr \& Wiese 1990). The $A_{k i}$-values taken as reference in this work have been labelled with an asterisk.

\begin{tabular}{ccc}
\hline \hline$\lambda(\mathrm{nm})$ & $\begin{array}{c}A_{k i}\left(10^{8} \mathrm{~s}^{-1}\right) \\
(\text { Fuhr \& Wiese 1990) }\end{array}$ & $\begin{array}{c}A_{k i}\left(10^{8} \mathrm{~s}^{-1}\right) \\
(\text { Castro et al. 2001) }\end{array}$ \\
\hline 457.720 & 0.960 & $0.831^{*}$ \\
458.285 & 0.760 & $0.812^{*}$ \\
461.528 & 0.540 & $0.509^{*}$ \\
461.915 & 0.810 & $0.748^{*}$ \\
481.176 & $0.170^{*}$ & \\
482.518 & 0.190 & $0.208^{*}$ \\
483.207 & 0.730 & $0.787^{*}$ \\
484.660 & & $0.762^{*}$ \\
530.866 & $0.024^{*}$ & \\
533.341 & & $0.500^{*}$ \\
\hline \hline
\end{tabular}

$\left(b=-1 / k T_{\mathrm{KrII}}^{\mathrm{exc}}\right)$ and the ordinate at null energy, it is possible to calculate the transition probabilities corresponding to all the measured lines, including those for which this value was previously known. In this method, the original set of data is assumed to be a good one as a whole, but the individual data may have uncertainties which sometimes are significant. This is the reason why both sets of data do not necessarily coincide. This explains the differences between the results from Castro et al. (2001) and those from Fuhr \& Wiese (1990) or those found between our results and those of Castro et al. (2001).

Temperature has also been obtained by the KrII/KrI intensities ratio in those instants where $\mathrm{KrI}$ and $\mathrm{KrII}$ lines with enough intensity could be recorded. Another method, based on the assumption that $N_{\mathrm{KrII}} / Z_{\mathrm{KrII}}=$ cte along the plasma life (Gigosos et al. 1994), was employed to calculate the temperature, $N_{\text {KrII }}$ being the KrII density and $Z_{\mathrm{KrII}}$ its partition function. The resulting three evolution curves are shown in Fig. 5. The very good agreement among these three methods, specially between the first two ones, seems to confirm that the plasma is well described by a pLTE model and is very near to total LTE. The quality of the linear fits and the agreement with temperatures obtained from the other methods allows us to estimate the $T_{\mathrm{KrII}}^{\mathrm{exc}}$ uncertainty lower than $10 \%$.

\section{Results and conclusions}

Once an $A_{k i}$-value has been obtained for each line and at each instant where this line was measured, plots representing all these values along the plasma life have been made. One of them, showing the measured transitions probabilities for the KrII 459.28 and $555.299 \mathrm{~nm}$ is shown in Fig. 6 . As can be seen, no systematic trends are observed in any of the lines. The random distribution around the mean value is a typical behaviour in these measurements. From now on, we will assign the mean value as the $A_{k i}$-value and the standard deviation as a quality indicator of the mean value. In this sense, it is significant that 28 of the 35

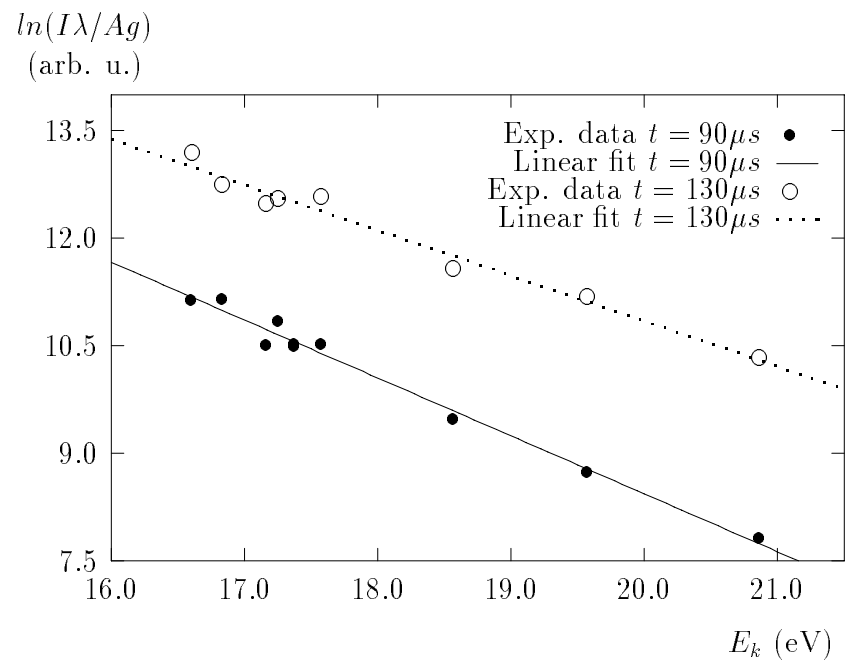

Fig. 4. Two examples of a Boltzmann plot performed in different instants of the plasma life. Population of excited states is plotted against the corresponding energy level.

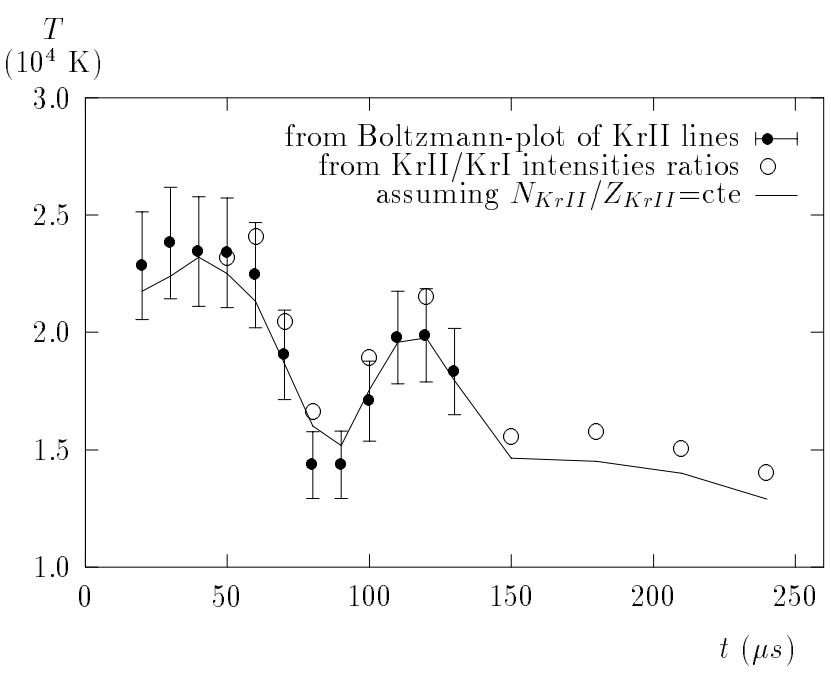

Fig. 5. Temperature evolution measured from Boltzmann-plot, from consecutive krypton intensities ratios and from the assumption $N_{\mathrm{KrII}} / Z_{\mathrm{KrII}}=$ cte.

measured KrII lines have uncertainties lower than $15 \%$ and only the 7 weakest lines show greater uncertainties. This result shows the quality of the measurements performed.

In Table 2, all measured transitions have been indicated. They have been ordered by increasing wavelength (first column). The second and third columns indicate the transition array and multiplet respectively, in all cases according to the notation suggested by Striganov \& Sventitskii (1968). The fourth and fifth columns contain all the experimental works considered, indicating in the case of data coming from this work the calculated standard deviation (in percentage) in parentheses. It is important to remark that, in the case of the work from Miller et al. (1972), this work offers relative transition probabilities but, since the value for $\lambda=435.5 \mathrm{~nm}$ is coincident with that recommended by Fuhr \& Wiese (1998), both 
Table 2. $A_{k i}$-values obtained in this work and the comparison with the existing literature. Next to the data from this work, statistical deviation in percentage is indicated in parentheses. Relative data from Miller et al. (1972) are rescaled to the value of Fuhr \& Wiese (1998) at $\lambda=435.5 \mathrm{~nm}$.

\begin{tabular}{|c|c|c|c|c|c|c|}
\hline $\begin{array}{c}\lambda \\
(\mathrm{nm})\end{array}$ & Transition & Multiplet & $\begin{array}{c}A_{k i} \\
\left(10^{8} \mathrm{~s}^{-1}\right)\end{array}$ & $\begin{array}{r}\text { Ref. } \\
(\exp )\end{array}$ & $\begin{array}{c}A_{k i} \\
\left(10^{8} \mathrm{~s}^{-1}\right)\end{array}$ & $\begin{array}{l}\text { Ref. } \\
\text { (th) }\end{array}$ \\
\hline \multirow[t]{11}{*}{457.720} & $5 \mathrm{~s}^{\prime}-5 \mathrm{p}^{\prime}$ & ${ }^{2} \mathrm{D}_{5 / 2}-{ }^{2} \mathrm{~F}_{7 / 2}^{\mathrm{o}}$ & $0.741(12)$ & This work & 1.415 & Spector \& Garpman (1977) \\
\hline & & & 6.9 & Levchenko (1971) & & \\
\hline & & & 1.54 & Miller et al. (1972) & & \\
\hline & & & 0.961 & Keil (1973) & & \\
\hline & & & 1.05 & Baessler et al. (1979) & & \\
\hline & & & 1.23 & Fonseca \& Campos (1982) & & \\
\hline & & & 0.795 & Brandt et al. (1982) & 1.15 & Brandt et al. (1982) \\
\hline & & & 2.76 & Bertuccelli \& Di Rocco (1991) & & \\
\hline & & & 0.73 & Samoilov et al. (1975) & & \\
\hline & & & 0.831 & Castro et al. (2001) & & \\
\hline & & & 0.96 & Fuhr \& Wiese (1998) & & \\
\hline \multirow[t]{3}{*}{458.285} & $5 p-6 s$ & ${ }^{4} \mathrm{D}_{5 / 2}^{\mathrm{o}}-{ }^{4} \mathrm{P}_{3 / 2}$ & $0.811(2)$ & This work & & \\
\hline & & & 0.812 & Castro et al. (2001) & & \\
\hline & & & 0.76 & Fuhr \& Wiese (1998) & & \\
\hline 459.280 & $5 \mathrm{p}^{\prime}-4 \mathrm{~d}^{\prime \prime}$ & ${ }^{2} \mathrm{P}_{3 / 2}^{\mathrm{o}}{ }^{2} \mathrm{D}_{5 / 2}$ & $0.293(6)$ & This work & & \\
\hline 459.849 & $5 \mathrm{p}-6 \mathrm{~s}$ & ${ }^{2} \mathrm{P}_{3 / 2}^{\mathrm{o}}-{ }^{4} \mathrm{P}_{1 / 2}$ & $0.232(9)$ & This work & & \\
\hline 460.402 & $5 p-6 s$ & ${ }^{4} \mathrm{D}_{1 / 2}^{\mathrm{o}}-{ }^{4} \mathrm{P}_{1 / 2}$ & $0.420(11)$ & This work & & \\
\hline \multirow[t]{7}{*}{461.528} & $5 s-5 p$ & ${ }^{2} \mathrm{P}_{3 / 2}{ }^{2} \mathrm{P}_{3 / 2}^{\mathrm{o}}$ & $0.499(2)$ & This work & 0.7898 & Koozekanani \& Trusty (1969) \\
\hline & & & 0.87 & Miller et al. (1972) & 0.732 & Spector \& Garpman (1977) \\
\hline & & & 0.99 & Podbiralina et al. (1973) & 0.125 & El Sherbini (1976) \\
\hline & & & 0.23 & Samoilov et al. (1975) & & \\
\hline & & & 1.55 & Bertuccelli \& Di Rocco (1991) & & \\
\hline & & & 0.509 & Castro et al. (2001) & & \\
\hline & & & 0.54 & Fuhr \& Wiese (1998) & & \\
\hline \multirow[t]{8}{*}{461.915} & $5 s-5 p$ & ${ }^{2} \mathrm{P}_{3 / 2}{ }^{-2} \mathrm{D}_{5 / 2}^{\mathrm{o}}$ & $0.74(12)$ & This work & 0.771 & Koozekanani \& Trusty (1969) \\
\hline & & & 1.47 & Miller et al. (1972) & 0.325 & El Sherbini (1976) \\
\hline & & & 0.808 & Keil (1973) & 1.24 & Spector \& Garpman (1977) \\
\hline & & & 0.45 & Samoilov et al. (1975) & & \\
\hline & & & 0.817 & Brandt et al. (1982) & 1.24 & Brandt et al. (1982) \\
\hline & & & 1.62 & Bertuccelli \& Di Rocco (1991) & & \\
\hline & & & 0.748 & Castro et al. (2001) & & \\
\hline & & & 0.81 & Fuhr \& Wiese (1998) & & \\
\hline 479.633 & $5 p-6 s$ & ${ }^{4} \mathrm{~S}_{3 / 2}^{\mathrm{o}}{ }^{2} \mathrm{P}_{1 / 2}$ & $0.180(9)$ & This work & & \\
\hline 480.297 & $4 d-5 p^{\prime}$ & ${ }^{4} \mathrm{P}_{5 / 2}{ }^{2} \mathrm{D}_{3 / 2}^{\mathrm{o}}$ & $0.006(12)$ & This work & & \\
\hline \multirow[t]{5}{*}{481.176} & $5 \mathrm{~s}-5 \mathrm{p}$ & ${ }^{4} \mathrm{P}_{1 / 2}-{ }^{4} \mathrm{D}_{3 / 2}^{\mathrm{o}}$ & $0.133(4)$ & This work & 0.412 & Koozekanani \& Trusty (1969) \\
\hline & & & 0.9 & Levchenko (1971) & 0.166 & Spector \& Garpman (1977) \\
\hline & & & 0.46 & Miller et al. (1972) & 0.005 & El Sherbini (1976) \\
\hline & & & 0.32 & Bertuccelli \& Di Rocco (1991) & & \\
\hline & & & 0.17 & Fuhr \& Wiese (1998) & & \\
\hline \multirow[t]{7}{*}{482.518} & $5 s-5 p$ & ${ }^{2} \mathrm{P}_{1 / 2}-{ }^{4} \mathrm{~S}_{3 / 2}^{\mathrm{o}}$ & $0.246(5)$ & This work & 0.073 & Koozekanani \& Trusty (1969) \\
\hline & & & 0.08 & Levchenko (1971) & 0.388 & Spector \& Garpman (1977) \\
\hline & & & 0.5 & Miller et al. (1972) & 0.153 & El Sherbini (1976) \\
\hline & & & 0.33 & Podbiralina et al. (1973) & & \\
\hline & & & 0.54 & Bertuccelli \& Di Rocco (1991) & & \\
\hline & & & 0.208 & Castro et al. (2001) & & \\
\hline & & & 0.19 & Fuhr \& Wiese (1998) & & \\
\hline
\end{tabular}

sets of data are comparable between them and of course, with our data. The sixth and seventh columns contain the $A_{k i}$-values obtained from theoretical calculations.

When taking a look at the comparisons among data in Table 2, the first noticeable point is the great scatter in the data. This is a very old problem in KrII transition probabilities, with two different aspects: the absolute scale of the $A_{k i}$-values compared and the quality of the relative ones. In this work, as explained in Sect. 3.3, the scale selected by us to transform our relative measurements to absolute ones corresponds to that of Castro et al. (2001), which is really the same as that of Fuhr \& Wiese (1998). In 
Table 2. continued.

\begin{tabular}{|c|c|c|c|c|c|c|}
\hline $\begin{array}{c}\lambda \\
(\mathrm{nm})\end{array}$ & Transition & Multiplet & $\begin{array}{c}A_{k i} \\
\left(10^{8} \mathrm{~s}^{-1}\right)\end{array}$ & $\begin{array}{r}\text { Ref. } \\
(\exp )\end{array}$ & $\begin{array}{c}A_{k i} \\
\left(10^{8} \mathrm{~s}^{-1}\right)\end{array}$ & $\begin{array}{l}\text { Ref } \\
\text { (th) }\end{array}$ \\
\hline 483.207 & $5 s-5 p$ & ${ }^{4} \mathrm{P}_{3 / 2}{ }^{-4} \mathrm{P}_{1 / 2}^{\mathrm{o}}$ & $\begin{array}{c}0.896(12) \\
4.98 \\
1.46 \\
1.67 \\
0.787 \\
0.73\end{array}$ & $\begin{array}{r}\text { This work } \\
\text { Levchenko (1971) } \\
\text { Miller et al. (1972) } \\
\text { Bertuccelli \& Di Rocco (1991) } \\
\text { Castro et al. (2001) } \\
\text { Fuhr \& Wiese (1998) }\end{array}$ & $\begin{array}{l}1.061 \\
1.127 \\
0.584\end{array}$ & $\begin{array}{r}\text { Koozekanani \& Trusty (1969) } \\
\text { Spector \& Garpman (1977) } \\
\text { El Sherbini (1976) }\end{array}$ \\
\hline 483.656 & $5 p-5 d$ & ${ }^{2} \mathrm{~S}_{1 / 2}^{\mathrm{o}}-{ }^{4} \mathrm{D}_{1 / 2}$ & $0.372(6)$ & This work & & \\
\hline 484.660 & $5 \mathrm{~s}-5 \mathrm{p}$ & ${ }^{2} \mathrm{P}_{3 / 2}{ }^{-2} \mathrm{P}_{1 / 2}^{\mathrm{o}}$ & $\begin{array}{c}0.796(9) \\
1.75 \\
2.54 \\
0.898 \\
2.4 \\
0.762\end{array}$ & $\begin{array}{r}\text { This work } \\
\text { Miller et al. (1972) } \\
\text { Podbiralina et al. (1973) } \\
\text { Brandt et al. (1982) } \\
\text { Bertuccelli \& Di Rocco (1991) } \\
\text { Castro et al. (2001) }\end{array}$ & $\begin{array}{c}<0.0009 \\
0.36\end{array}$ & $\begin{array}{l}\text { El Sherbini (1976) } \\
\text { Brandt et al. (1982) }\end{array}$ \\
\hline 530.866 & $5 \mathrm{~s}-5 \mathrm{p}$ & ${ }^{4} \mathrm{P}_{3 / 2}-{ }^{4} \mathrm{P}_{5 / 2}^{\mathrm{o}}$ & $\begin{array}{c}0.025(5) \\
0.024\end{array}$ & $\begin{array}{r}\text { This work } \\
\text { Fuhr \& Wiese (1998) }\end{array}$ & $\begin{array}{l}0.043 \\
0.071\end{array}$ & $\begin{array}{r}\text { Koozekanani \& Trusty (1969) } \\
\text { El Sherbini (1976) }\end{array}$ \\
\hline 531.741 & $5 p-5 d$ & ${ }^{2} \mathrm{D}_{3 / 2}^{\mathrm{o}}{ }^{-4} \mathrm{P}_{1 / 2}$ & $0.038(9)$ & This work & & \\
\hline 532.277 & $5 p-6 s$ & ${ }^{2} \mathrm{P}_{1 / 2}^{\mathrm{o}}-{ }^{4} \mathrm{P}_{3 / 2}$ & $0.042(5)$ & This work & & \\
\hline 533.341 & $4 d^{\prime}-5 f$ & ${ }^{2} \mathrm{D}_{5 / 2}-{ }^{2} \mathrm{~F}_{7 / 2}^{\mathrm{o}}$ & $\begin{array}{c}0.494(1) \\
0.49\end{array}$ & $\begin{array}{r}\text { This work } \\
\text { Castro et al. (2001) }\end{array}$ & & \\
\hline 534.676 & $5 p-6 s$ & ${ }^{4} \mathrm{D}_{3 / 2}^{\mathrm{o}}-{ }^{4} \mathrm{P}_{5 / 2}$ & $0.028(6)$ & This work & & \\
\hline 535.545 & $4 d-5 f$ & ${ }^{2} \mathrm{D}_{5 / 2}-{ }^{4} \mathrm{~F}_{5 / 2}^{\mathrm{o}}$ & $0.018(9)$ & This work & & \\
\hline 541.843 & $4 d^{\prime}-5 f$ & ${ }^{2} \mathrm{D}_{3 / 2}{ }^{-2} \mathrm{D}_{3 / 2}^{\mathrm{o}}$ & $0.079(16)$ & This work & & \\
\hline 543.863 & $4 d-5 p$ & ${ }^{4} \mathrm{D}_{1 / 2}-{ }^{4} \mathrm{D}_{1 / 2}^{\mathrm{o}}$ & $0.058(29)$ & This work & & \\
\hline 544.634 & $4 d-5 p$ & ${ }^{4} \mathrm{D}_{1 / 2}-{ }^{2} \mathrm{P}_{3 / 2}^{\mathrm{o}}$ & $0.027(9)$ & This work & & \\
\hline 546.817 & $4 d^{\prime}-5 f$ & ${ }^{2} \mathrm{D}_{3 / 2}-{ }^{2} \mathrm{~F}_{5 / 2}^{\mathrm{o}}$ & $0.332(13)$ & This work & & \\
\hline 549.954 & $5 \mathrm{~s}-5 \mathrm{p}$ & ${ }^{4} \mathrm{P}_{1 / 2}-{ }^{4} \mathrm{P}_{1 / 2}^{\mathrm{o}}$ & $0.014(11)$ & This work & $\begin{array}{l}0.030 \\
0.021\end{array}$ & $\begin{array}{r}\text { Koozekanani \& Trusty (1969) } \\
\text { El Sherbini (1976) }\end{array}$ \\
\hline 553.229 & $4 d-5 p^{\prime}$ & ${ }^{2} \mathrm{~F}_{7 / 2}-{ }^{2} \mathrm{~F}_{7 / 2}^{\mathrm{o}}$ & $0.001(20)$ & This work & & \\
\hline 555.299 & $4 d^{\prime}-5 f$ & ${ }^{2} \mathrm{D}_{3 / 2}-{ }^{4} \mathrm{~F}_{5 / 2}^{\mathrm{o}}$ & $0.109(6)$ & This work & & \\
\hline 556.865 & $4 d-5 p$ & ${ }^{4} \mathrm{D}_{5 / 2}-{ }^{4} \mathrm{D}_{3 / 2}^{\mathrm{o}}$ & $\begin{array}{l}0.034(3) \\
0.025 \\
0.025\end{array}$ & $\begin{array}{r}\text { This work } \\
\text { Podbiralina et al. (1973) } \\
\text { Samoilov et al. }(1975)\end{array}$ & & \\
\hline 565.037 & $4 d-5 p^{\prime}$ & ${ }^{2} \mathrm{P}_{3 / 2}{ }^{-2} \mathrm{D}_{5 / 2}^{\mathrm{o}}$ & $0.006(38)$ & This work & & \\
\hline 568.189 & $5 s-5 p$ & ${ }^{2} \mathrm{P}_{3 / 2}-{ }^{4} \mathrm{D}_{5 / 2}^{\mathrm{o}}$ & $0.100(8)$ & This work & $\begin{array}{l}0.314 \\
0.358\end{array}$ & $\begin{array}{r}\text { Koozekanani \& Trusty (1969) } \\
\text { El Sherbini (1976) }\end{array}$ \\
\hline 569.035 & $4 d-5 p^{\prime}$ & ${ }^{2} \mathrm{P}_{3 / 2}{ }^{-2} \mathrm{D}_{3 / 2}^{\mathrm{o}}$ & $\begin{array}{c}0.246(7) \\
0.082\end{array}$ & $\begin{array}{r}\text { This work } \\
\text { Samoilov et al. (1975) }\end{array}$ & & \\
\hline 574.927 & $5 p^{\prime}-5 d$ & ${ }^{2} \mathrm{D}_{5 / 2}^{\mathrm{o}}{ }^{-2} \mathrm{D}_{5 / 2}$ & $0.018(36)$ & This work & & \\
\hline 575.298 & $5 \mathrm{~s}-5 \mathrm{p}$ & ${ }^{2} \mathrm{P}_{1 / 2}-{ }^{4} \mathrm{D}_{3 / 2}^{\mathrm{o}}$ & $0.014(8)$ & This work & $\begin{array}{l}0.037 \\
0.013\end{array}$ & $\begin{array}{r}\text { Koozekanani \& Trusty (1969) } \\
\text { El Sherbini (1976) }\end{array}$ \\
\hline $\begin{array}{l}577.141 \\
577.772\end{array}$ & $\begin{array}{l}4 d-5 p \\
4 d^{\prime}-5 f\end{array}$ & $\begin{array}{l}{ }^{4} \mathrm{D}_{1 / 2}-{ }^{2} \mathrm{P}_{1 / 2}^{\mathrm{o}} \\
{ }^{2} \mathrm{~F}_{5 / 2}-{ }^{4} \mathrm{~F}_{7 / 2}^{\mathrm{o}}\end{array}$ & $\begin{array}{c}0.086(8) \\
0.020(39)\end{array}$ & $\begin{array}{l}\text { This work } \\
\text { This work }\end{array}$ & & \\
\hline
\end{tabular}

this last publication, the authors maintain as a reference the same data published by NIST from 1978 (Wiese \& Martin 1978, 1980; Fuhr \& Wiese 1990, 1996). If we reexamine Fig. 4, we see the nice linear behaviour of the KrII excited states population, a situation always present with our plasma source (e.g. Gigosos et al. 1994; Aparicio et al. 1997; del Val et al. 2000; Mar et al. 2000) and a first result can be guessed. The data from Castro et al. (2001), from Fuhr \& Wiese (1998) and therefore, from ourselves, might not be a good absolute scale, but at least does seem to represent a good relative one. Small differences between the data from Castro et al. (2001) and ours, corresponding to measurements performed in the same plasma source, arise from the uncertainty in intensity measurements and statistical deviations of the fits in the Boltzmann plots.

If we compare our data with other experimental ones, we find a curious agreement with those from Brandt et al. (1982) and Keil (1973). Both works correspond to measurements performed in wall-stabilized arcs at atmospheric pressure by assuming total LTE. Although there are only three data points to compare with Brandt et al. (1982), the mean ratio between our data and theirs is 0.91 


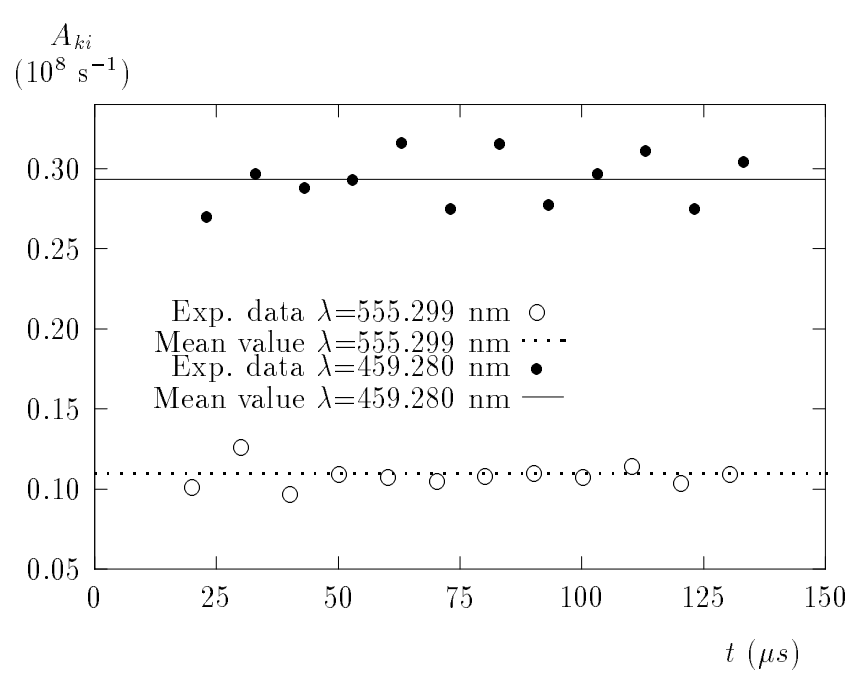

Fig. 6. Two examples of the evolution of the measured transition probabilities measured in this work along the plasma life. No systematic trends are detected.

with only $2 \%$ statistical deviation. The arc at atmospheric pressure is probably one of the plasma sources closest to LTE and the two-wavelength interferometry technique employed by them in $N_{\mathrm{e}}$ diagnostics is one of the most accurate ones used to determine this parameter. The plasma generated in our work has been demonstrated to be well described by a pLTE model and other plasmas generated by this source have been shown to be very close to LTE (Aparicio et al. 1999). As a conclusion, we can say that the scale from Brandt et al. (1982) is probably near to the absolute scale. In relation to data from Miller et al. (1972), very frequently considered as a good reference in a relative scale, we do not find good coincidence with our data. In fact, even assuming that $\lambda=435.5 \mathrm{~nm}$ is not a good line to use as the reference to rescale their work (this is one of the most prominent lines in KrII visible spectra and is very sensitive to self-absorption), we find that the ratio between their data and ours is $2.11 \pm 25 \%$. We can conclude that data from Miller et al. (1972) must be taken with care as a relative scale of KrII transition probabilities. These comparisons can be shown in Fig. 7 . If we try comparisons with Bertuccelli \& Di Rocco (1991), the mean ratio between their data and ours is $2.64 \pm 23 \%$. Comparisons with other experimental works reveal greater differences between their relative scales and ours.

Relative to theoretical works, comparisons with data from Koozekanani \& Trusty (1969), based on intermediate coupling calculations with the absolute values obtained from Hartree-Fock functions, are very poor. Their data are on average 1.87 times greater than ours, with a deviation around $49 \%$. Discrepancies are even greater when compared to calculations from El Sherbini (1976). However, we note the very good coincidence between the conclusions extracted by Brandt et al. (1982) and ours in relation to calculations performed by Spector \& Garpman (1977), on the basis of intermediate coupling coefficients with the radial integral obtained from relativistic self-consistent-field

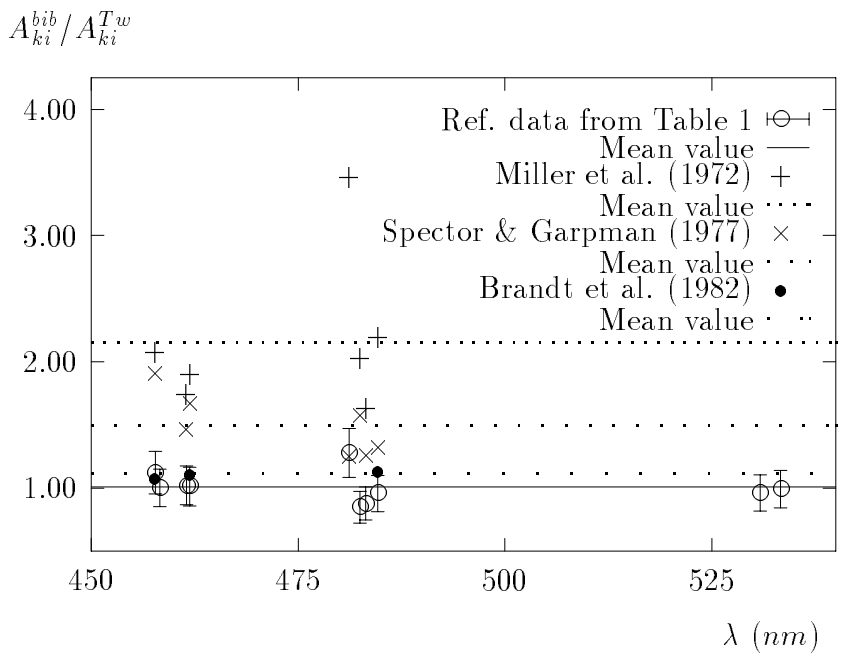

Fig. 7. Comparisons between the ratios of data taken from the bibliography (bib) and ours (Tw). Data taken from Table 1 correspond with those labelled with an asterisk there, that is to say, those employed in the Boltzmann plots. For the ratios with these data, $15 \%$ error bars have been considered for our results.

wavefunctions. They found a mean ratio between their data and those from this theoretical reference of around $1.5 \pm 14 \%$. Certainly, the present work contains only three lines measured by Brandt et al. (1982) as stated before, and four other ones not measured by them but calculated by Spector \& Garpman (1977), but curiously the mean ratio between this theoretical work and our data is $1.493 \pm 15 \%$, almost the same as that found by Brandt et al. (1982) with a systematic but reasonable deviation. We think this result reinforces the idea of a good placing of our data in a relative scale and is probably not far from an absolute one.

As a final conclusion, this work offers transition probabilities of a set of $35 \mathrm{KrII}$ lines in the spectral region from 450 to $580 \mathrm{~nm}$. For 20 of them, there are no previous data. We can estimate an error of $15 \%$ for more than $80 \%$ of them and $40 \%$ for the rest, always on a relative scale. Many of them will be useful in refining new calculation models. Furthermore, this work sheds some light what are probably the most significant theoretical and experimental works, those of Spector \& Garpman (1977) and Brandt et al. (1982) respectively. However, new and more precise calculations and measurements are still required in order to clarify the uncertainties remaining in KrII transition probabilities.

Acknowledgements. The authors thank S. González for his collaboration in the experimental arrangement, and the Spanish Dirección General de Investigación Científica y Técnica (Ministerio de Educación y Ciencia) and the Consejería de Educación y Cultura de Castilla y León for their financial support under contracts No. PB98-0356 and VA23-99 respectively. Dr. J. A. Aparicio wants to express his personal acknowledgement to the Organización Nacional de Ciegos de España (ONCE) for help. 


\section{References}

Aparicio, J. A., Gigosos, M. A., \& Mar, S. 1997, J. Phys. B, 30,3141

Aparicio, J. A., Gigosos, M. A., González, V. R., et al. 1998, J. Phys. B, 31, 1029

Aparicio, J. A., del Val, J. A., González, V. R., et al. 1999, J. Phys. Soc. Jpn., 68, 3885

Baessler, P., Obbarius, H.-U., \& Schulz-Gulde, E. 1979, Physica C, 96, 147

Bertuccelli, G., \& Di Rocco, H. O., Spectrosc. Lett., 24, 1039

Brandt, T., Helbig, V., \& Nick, K.-P. 1982, J. Phys. B, 15, 2139

Cardelli, J. A., Sarage, B. D., \& Ebbets, D. C. 1991, ApJL, 383,23

Cardelli, J. A., \& Mayer, S. 1997, ApJL, 477, 57

de Castro, A., Aparicio, J. A., del Val, J. A., González, V. R., \& Mar, S. 2001, J. Phys. B, submitted

Donnelly, K. E., Kindlmann, P. J., \& Bennett, Jr. W. R. 1975, J. Opt. Soc. Am., 65, 1359

Fonseca, V., \& Campos, J. 1982, J. Phys. B, 15, 2349

El Sherbini, Th. M. 1976, Z. Phys. A, 276, 325

Fuhr, J. R., \& Wiese, W. L. 1990, CRC Handbook of Chemistry and Physics, ed. D. R. Lide (CRC Press. Inc., Boca Raton, Florida), 71th edition, 128

Fuhr, J. R., \& Wiese, W. L. 1996, CRC Handbook of Chemistry and Physics, ed. D. R. Lide, \& H. P. R. Frederikse (CRC Press. Inc., Boca Raton, Florida), 77th edition, 128

Fuhr, J. R., \& Wiese, W. L. 1998, CRC Handbook of Chemistry and Physics, ed. D. R. Lide (CRC Press. Inc., Boca Raton, Florida), 78th edition, 154

Gigosos, M. A., Mar, S., Pérez, C., \& de la Rosa, I. 1994, Phys. Rev. E, 49, 1575

González, V. R. 1999, Medida de parámetros atómicos en un plasma de silicio, Ph.D. Thesis, Univ. Valladolid

González, V. R., Aparicio, J. A., del Val, J. A., \& Mar, S. 2000, A\&A, 363, 1177
Graves, J. F. 1983, Proceedings of the International Symposiumon on Micoelectronics, 147

Keil, H. W. 1973, Diplomarbeit Univ. Kiel

Koozekanani, S. H., \& Trusty, G. L. 1969, J. Opt. Soc. Am., 59,1281

Leckrone, D. S., et al. 1993, Peculiar Versus Normal Phenomena in A-type and Related Stars, ed. M. Dworestski, F. Castelli, \& R. Faraggiana, ASP Conf. Ser. 44 (Astronomical Society of the Pacific, San Francisco), 42 Levchenko, M. A. 1971, Sov. Phys. J., 14, 1445

Mar, S., Pérez, C., González, V. R., et al. 2000, A\&AS, 144, 509

Mckee, J. L. J., Toth, W. D., \& Fath, P. M. 1996, Proceedings of the International Symposiumon on Microelectronics, 259

Miller, M. H., Roig, R. A., \& Bengtson, R. D. 1972, J. Opt. Soc. Am., 62, 1027

van der Mullen, J. A. M. 1990, Phys. Rep., 191, 109

Podbiralina, V. P., Smirnov, Yu. M., \& Stegnova, N. V. 1973, Opt. Spectrosc., 34, 467

de la Rosa, M. I., Pérez, C., de Frutos, A. M., \& Mar, S. 1990, Phys. Rev. A, 42, 7389

Samoilov, V. P., Smirnov, Yu. M., \& Starikova, G. S. 1975, J. Appl. Spectrosc., 23, 1117

Spector, N., \& Garpman, S. 1977, J. Opt. Soc. Am., 67, 155

Striganov, A. R., \& Sventitskii, N. S. 1968, in Tables of Spectral of Neutral and Ionised Atoms (IF1/Plenum, New York)

del Val, J. A., Mar, S., Gigosos, M. A., de la Rosa, I., Pérez, C., \& González, V. R. 1998, Jpn. J. Appl. Phys., 37, 4177

del Val, J. A., Aparicio, J. A., González, V., \& Mar, S. 2000, A\&A, 357, 1137

Wiese, W. L., \& Martin, G. A. 1978, CRC Handbook of Chemistry and Physics, ed. R. C. Weast, \& M. J. Astle (CRC Press. Inc., Florida), 59th edition, D112

Wiese, W. L., \& Martin, G. A. 1980, Wavelengths and Transition Probabilities for Atoms and Atomic Ions, Nat. Stand. Ref. Data Ser., Nat. Bur. Stand. (U.S.), 68, 359 